\title{
Role of Kainate Autoreceptors in Short-Term Plasticity at Hippocampal Mossy Fiber Synapses
}

\author{
Sheila L. Dargan ${ }^{1}$ and Mascia Amici ${ }^{2}$ \\ Department of ${ }^{1}$ Physiology and Pharmacology and ${ }^{2}$ Anatomy, Medical Research Council Centre for Synaptic Plasticity, University of Bristol, Bristol BS8 \\ 1TD, United Kingdom \\ Review of Scott et al. (http://www.jneurosci.org/cgi/content/full/28/49/13139)
}

Long-term potentiation (LTP) is a well established experimental model used to investigate the synaptic basis of learning and memory. Synapses formed by giant mossy fiber (MF) boutons onto CA3 pyramidal cells (PC) in the hippocampus are particularly interesting, because they show NMDA receptor-independent LTP and exhibit unusual short-term facilitation. Although presynaptic kainate receptors (KARs) are believed to play an essential role at MF-PC synapses, the mechanisms underlying LTP induction, frequency facilitation and calcium signaling remain poorly understood and controversial.

An interesting aspect of hippocampal MFs is that they contain multiple terminal types that synapse onto distinct target cells. Giant boutons $(4-8 \mu \mathrm{m})$ synapse onto hilar mossy cells and CA3 pyramidal cells, whereas smaller en passant terminals (1-2 $\mu \mathrm{m})$ and filopodia emanating from giant boutons synapse onto interneurons (IN). Short-term plasticity in MF terminals has been shown to be target-cell dependent. For example, use-dependent facilitation of glutamate release is significantly more pronounced at MF-PC synapses compared with MF-IN synapses

Received Feb. 2, 2009; revised March 18, 2009; accepted March 19, 2009. We thank Dr. Stephen Fitzjohn for discussion and comments.

Correspondence should be addressed to Dr. Sheila L. Dargan, Department of Physiology and Pharmacology, Medical Research Council Center for Synaptic Plasticity, School of Medical Sciences, University of Bristol, Bristol BS8 1TD, UK. E-mail: Sheila.Dargan@bristol.ac.uk.

DOI:10.1523/JNEUROSCI.0549-09.2009

Copyright $\odot$ 2009 Society for Neuroscience $\quad 0270-6474 / 09 / 295713-03 \$ 15.00 / 0$ in the CA3 subfield (Toth et al., 2000). Target-cell-dependent plasticity has important consequences because it allows slight increases in stimulation frequency to shift the balance from disynaptic inhibition to monosynaptic excitation. This phenomenon occurs during normal behavior; for example, when hippocampal neurons burst at high frequency as animals enter place-cell fields (Leutgeb et al., 2007). Pathological changes in this neuronal circuitry can disrupt the normal inhibition-excitation balance resulting in disorders such as epilepsy.

Until now, it has been widely agreed that KARs are involved in frequency facilitation and LTP at MF-PC synapses, but the subunit composition of these KARs (Jane et al., 2009) and the proposed role for intracellular calcium stores (Lauri et al., 2003) remain controversial. An article published recently in The Journal of Neuroscience by Scott et al. (2008) investigated the role of presynaptic KARs and intracellular calcium stores in short-term plasticity, using a relatively novel technique that combines two-photon microscopy, patch-clamp electrophysiology and presynaptic calcium imaging to record calcium dynamics in individual MF boutons (Fig. 1). The authors elegantly showed that presynaptic kainate autoreceptors and calcium stores are involved in shortterm plasticity at MF-PC synapses. Facilitation of presynaptic calcium transients in giant MF boutons was significantly reduced after KAR antagonism (with NBQX or UBP302) or calcium store blockade with ryanodine [Scott et al. (2008), their Fig. 1]. In contrast, calcium transients recorded from en passant boutons and hilar giant boutons were not affected by the same pharmacological manipulations [Scott et al. (2008), their Fig. 4]. In patchclamp recordings from CA3 neurons themselves, UBP302 also reduced pairedpulse facilitation of EPSCs, evoked by minimal MF stimulation, in pyramidal cells but not in mossy cells or interneurons [Scott et al. (2008), their Fig. 6]. In conclusion, they provide compelling evidence that presynaptic kainate autoreceptors contribute to short-term plasticity at MF-PC synapses, but not at other MF synapses.

In conflict with the findings of Scott et al. (2008), another recent investigation of excitatory hippocampal MF synapses concluded that presynaptic KARs do not play a role in short-term facilitation at MF-PC synapses (Kwon and Castillo, 2008). Recording electrophysiological responses from MF synapses in hippocampal brain slices is no trivial matter because electrical stimulation in the dentate gyrus can additionally activate contaminating non-MF inputs. Kwon and Castillo (2008) avoided such problems by recording MFmediated NMDA receptor currents in CA3 pyramidal cells, holding the cell at a positive potential and recording in the presence of an AMPA receptor antagonist. Using this approach, the authors found no evidence for presynaptic KAR activation by endogenous glutamate at stimula- 
tion frequencies up to $25 \mathrm{~Hz}$ and suggest that actions generally attributed to presynaptic KARs are likely caused by effects on recurrent CA3 network activity. Moreover, Kwon and Castillo (2008) did not observe facilitation of NMDA receptor currents in response to bath application of kainate, whereas others have done so under similar recording conditions (Schmitz et al., 2001; Bortolotto et al., 2003). Although it is currently not possible to explain this latter discrepancy, recurrent CA3 activity is unlikely the explanation for why Scott et al. (2008) observe KAR-mediated facilitation, because they used very minimal stimulation protocols (injecting current into individual labeled granule cells to induce action potential-evoked calcium entry or stimulating single mossy fibers to evoke postsynaptic responses).

Controversy surrounding the role of presynaptic KARs in short-term facilitation at MF-PC synapses may arise for several reasons, not least the experimental preparation. MFs synapse onto several distinct target cells, so bath application of pharmacological agents to investigate receptor-mediated mechanisms at one type of synapse may additionally alter activity at synapses with other target cells. Furthermore, care must be taken when preparing brain slices to ensure the MF projection from dentate gyrus to CA3 remains intact. Another, less investigated possibility is that the axons stimulated in classical MF slice electrophysiological experiments may actually arise from different populations of cells with different properties, such as newborn granule cells or semilunar granule cells in the inner molecular layer (Williams et al., 2007). The technique of Scott et al. (2008) overcomes all of these issues because it involves labeling individual granule cells, tracing their single MFs into the CA3 region and making high resolution recordings from visually identified boutons. Although field-potential and whole-cell electrophysiological recordings are invaluable tools for probing the mechanisms that underlie synaptic plasticity, such recordings represent an integrated response, therefore masking variation at individual synapses. The only definitive way to show that MF KAR-mediated facilitation induces synaptic changes in the postsynaptic CA3 pyramidal cell would be to make paired recordings in acute slices, ideally combining calcium imaging and electrophysiology. However, these experiments are technically difficult because of the sparse connectivity between these cell types.

Although the aim of the Scott et al.

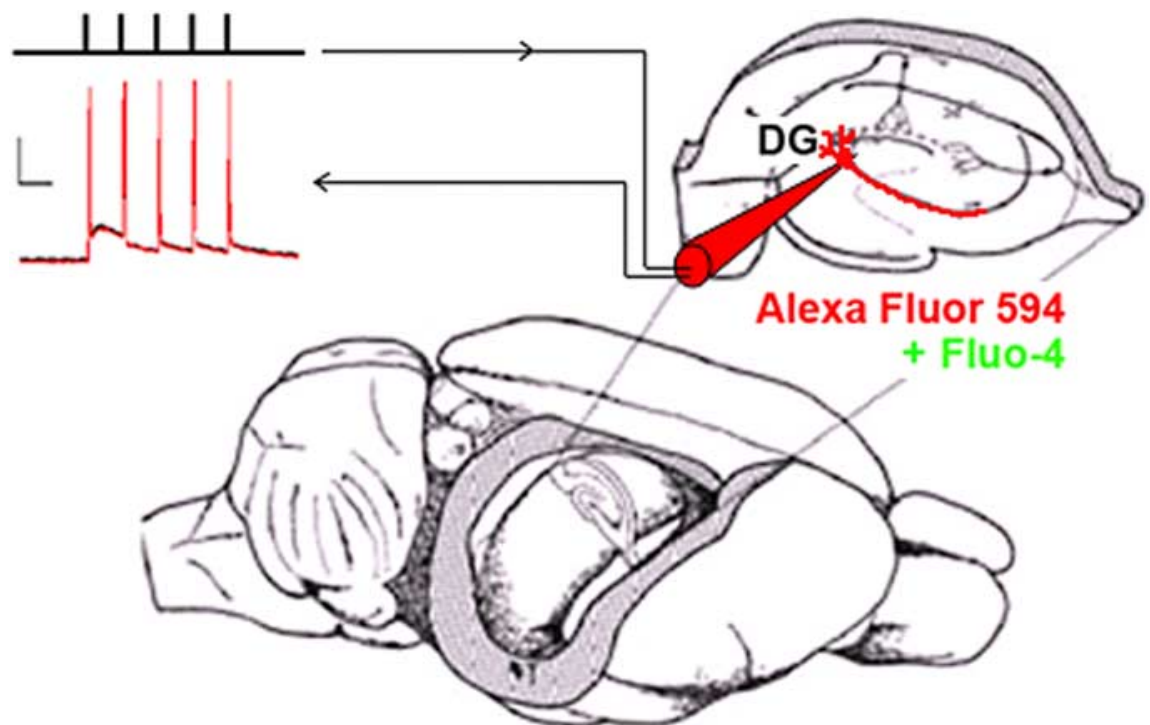

Figure 1. Presynaptic calcium imaging in individual mossy fiber boutons. Schematic of technique used by Scott et al. (2008). Whole-cell patch-clamp recordings are made from granule cells of dentate gyrus (DG) in acute slices. Patch pipettes contain a red morphological marker (Alexa Fluor 594), to allow the axon to be traced into CA3 region of hippocampus, together with a calcium indicator dye (Fluo-4). Calcium transients are recorded in selected boutons in response to current injection via the patch pipette. Calibration: $50 \mathrm{mV}$, (vertical), $50 \mathrm{~ms}$ (horizontal).

(2008) study was not to address the controversy as to which KAR subunits are involved in short-term plasticity at MF-PC synapses, their data (UBP302) [Scott et al. (2008), their Fig. 1] do appear to support a functional role for presynaptic GluK1. More recently, it has been shown that another potent GluK1-selective antagonist, ACET, also reduces presynaptic calcium entry into giant MF boutons, and reversibly blocks the induction of MF-LTP (Dargan et al., 2009). However, the findings of a study published at the same time do not appear to support a role for GluK1containing KARs in short-term facilitation at MF-PC synapses, instead implicating the involvement of GluK2/GluK3 heteromers (Perrais et al., 2009). Moreover, this study shows that the "GluK1selective" antagonists UBP302 and ACET may also act at the GluK3 subunit (Perrais et al., 2009). Although such apparent controversies remain difficult to resolve at the current time, the development of novel KAR subunit-selective antagonists combined with direct recordings from single granule cells and individual boutons will undoubtedly help.

In light of the current controversies, the results presented in the Scott et al. (2008) study are immensely insightful. Presynaptic functions of KARs have generally been inferred from experiments where multiple axons are stimulated, which, as discussed by the authors, "may conceal variability among synapses, and also fail to distinguish between spillover- mediated interactions among synapses and a possible autoreceptor function in the strict sense." The elegance of the presynaptic calcium imaging technique resides in the fact that patching an individual granule cell, tracing its axon into the $\mathrm{CA} 3$ region, and stimulating by current injection via the patch pipette, can leave no doubt that these are pure MF recordings. The findings of Scott et al. (2008) provide the most direct evidence to date that presynaptic kainate receptors are present on giant mossy fiber terminals where they act as true autoreceptors, responding to glutamate released from the same terminal as that on which they reside. Moreover, an unambiguous role for presynaptic KARs coupled to intracellular calcium stores is shown to exist in giant MF terminals.

\section{References}

Bortolotto ZA, Lauri S, Isaac JT, Collingridge GL (2003) Kainate receptors and the induction of mossy fibre long-term potentiation. Philos Trans R Lond B Biol Sci: 358:657-666.

Dargan SL, Clarke VR, Alushin GM, Sherwood JL, Nisticò R, Bortolotto ZA, Ogden AM, Bleakman D, Doherty AJ, Lodge D, Mayer ML, Fitzjohn SM, Jane DE, Collingridge GL (2009) ACET is a highly potent and specific kainate receptor antagonist: characterisation and effects on hippocampal mossy fibre function. Neuropharmacology 56:121-130.

Jane DE, Lodge D, Collingridge GL (2009) Kainate receptors: pharmacology, function and therapeutic potential. Neuropharmacology 56:90-113.

Kwon HB, Castillo PE (2008) Role of glutamate 
autoreceptors at hippocampal mossy fiber synapses. Neuron 60:1082-1094.

Lauri SE, Bortolotto ZA, Nistico R, Bleakman D, Ornstein PL, Lodge D, Isaac JT, Collingridge GL (2003) A role for $\mathrm{Ca}^{2+}$ stores in kainate receptor-dependent synaptic facilitation and LTP at mossy fiber synapses in the hippocampus. Neuron 39:327-341.

Leutgeb JK, Leutgeb S, Moser MB, Moser EI (2007) Pattern separation in the dentate gyrus and CA3 of the hippocampus. Science 315:961-966.
Perrais D, Pinheiro PS, Jane DE, Mulle C (2009) Antagonism of recombinant and native GluK3-containing kainate receptors. Neuropharmacology 56:131-140.

Schmitz D, Mellor J, Nicoll RA (2001) Presynaptic kainate receptor mediation of frequency facilitation at hippocampal mossy fiber synapses. Science 291:1972-1976.

Scott R, Lalic T, Kullmann DM, Capogna M, Rusakov DA (2008) Target-cell specificity of kainate autoreceptor and $\mathrm{Ca}^{2+}$-storedependent short-term plasticity at hippocam- pal mossy fiber synapses. J Neurosci 28:13139-13149.

Toth K, Suares G, Lawrence JJ, Philips-Tansey E, McBain CJ (2000) Differential mechanisms of transmission at three types of mossy fiber synapse. J Neurosci 20:82798289.

Williams PA, Larimer P, Gao Y, Strowbridge BW (2007) Semilunar granule cells: glutamatergic neurons in the rat dentate gyrus with axon collaterals in the inner molecular layer. J Neurosci 27:13756-13761. 